\title{
4 Integral Domains
}

In this chapter we introduce some properties that arise from integral domains. We provide lemmas and propositions in order the structures to be better understood. For this chapter $V$ is an integral domain and $F$ the field of fractions of $V$.

\subsection{Fractional Ideals}

A very interesting structure that is highly connected to the Dedekind domains are the fractional ideals.

Definition Let $V$ be an integral domain and $F$ its field of fractions. The $V$-module $I$ of $F$ is called a fractional ideal for $V$ if there exists a non-zero $a \in V$ such that $a I \subset V$.

It is easy to see that for $a=1$ the fractional ideal $I$ coincides to the usual ideal often referred as the integral ideal in order to be distinguished from the arbitrary fractional ideal.

Next, we define the ordinary operations among fractional ideals as usual. That is, the sum of two ideals $I$ and $J$ is the set that consists of all the elements $a+b$ where $a$ and $b$ are elements of $I$ and $J$ respectively. The set of all finite sums

$$
\sum a_{i} b_{j}, \text { where } a_{i} \in I, b_{j} \in J
$$

is the product of $I$ and $J$. Finally, the quotient of $I$ by $J$ is the set

$$
\{a \in F \mid a J \subseteq I\}
$$

and is denoted by $(I: J)$. Obviously, for $I=V$ we get that $(V: J)=J^{-1}$.

Definition Let $I$ be a fractional ideal of $V$. If there is a fractional ideal $J$ such that $I J=V$ then $I$ is called invertible.

Lemma 4.1.1. Let $I$ be a fractional ideal of $V$. If $J$ is a fractional ideal of $V$ such that $I J=V$, then $J=I^{-1}$.

Proof. It is enough to show that $J$ is $(V: I)$. We have that

$$
J \subset(V: I)=(V: I) \cdot V=(V: I) \cdot I \cdot J \subset V \cdot J=J .
$$

The result follows.

Lemma 4.1.2. Every invertible ideal is finitely generated 
Proof. Let $I$ be an invertible ideal of $V$. Since $I I^{-1}=V$ it implies that there are $a_{i} \in I$ and $a_{i}^{\prime} \in I^{-1}$ such that

$$
a_{1} a_{1}^{\prime}+\cdots+a_{n} a_{n}^{\prime}=1
$$

By multiplying the above equation with $b \in I$ we get

$$
b a_{1} a_{1}^{\prime}+\cdots+b a_{n} a_{n}^{\prime}=b
$$

where $b a_{i}^{\prime}$ belong to $V$. Hence

$$
I=V a_{1}+\cdots+V a_{n}
$$

and therefore $\left\{a_{1}, \ldots, a_{n}\right\}$ are the generators of $I$.

Proposition 4.1.3. If every non-zero fractional ideal of an integral domain $V$ is invertible then $V$ is a Dedekind domain.

Proof. In order to prove that $V$ is a Dedekind domain we need to prove that $V$ is Noetherian, integrally closed and every prime ideal is maximal. Initially by 1.1.9 and 4.1.1 we deduce that $V$ is Noetherian.

Now let $a \in F$ integral over $V$. In order to prove that $V$ is integrally closed it is enough to prove that $V[a]=V$. Since every ideal of $V$ is invertible it follows that $V[a]$ is also invertible. Therefore there is $(V[a])^{-1}$ such that

$$
(V[a])^{-1} V[a]=V .
$$

Thus we have that

$$
V[a]=V[a] \cdot V \Rightarrow V[a]=V[a] \cdot V[a] \cdot(V[a])^{-1} .
$$

Since $V[a] \cdot V[a]=V[a]$ we have that

$$
V[a]=V[a] \cdot(V[a])^{-1} \Rightarrow V[a]=V
$$

which means that $V$ is integrally closed.

Finally let $I$ be a prime ideal and $P$ be maximal such that $I \subset P$. So, $I P^{-1}$ is a proper ideal and $\left(I P^{-1}\right) P=I$ which implies that $I P^{-1} \subset I$ or $P \subset I$ and therefore $I$ is equal to the maximal ideal $P$.

Definition Let $I \subseteq F$ be a fractional ideal of $V$. The set

$$
\operatorname{Ann}(I):=\{a \in F \mid a I=0\}
$$

is called annihilator of $I$.

The following lemma provides us with some basic properties of the annihilator of the quotient ideals. 
Lemma 4.1.4. Let $I$, $J$ be two fractional ideals of $V$. It holds that

(i) $\operatorname{Ann}(I+J)=A n n(I) \cap A n n(J)$.

(ii) $(I: J)=\operatorname{Ann}((I+J) / I)$.

(iii) If $I \cong V / J$ then Ann $(I)=J$.

Proof. (i). Let $a \in A n n(I) \cap A n n(J)$. Then, $a I=0$ and $a J=0$ which implies that $a(I+J)=0$. Thus, $a \in A n n(I+J)$.

Conversely, consider $a \in A n n(I+J)$. Then, by definition, $a \in F$ with $a(I+J)=0$ for every $c \in I$ and $b \in J$. Thus $\forall c \in I$ and $\forall b \in J$ we obtain that $a I=0$ and $a J=0$. The result follows.

(ii). Let $a \in A n n(I+J) / I$. Then, by definition, $a \in F$ with $a(I+J) \subseteq I$. So, $\forall b \in J$ we have that $a J \subseteq I$. Thus, $a \in(I: J)$.

Conversely, let $a \in(I: J)$, then $a \in F$ with $a J \subset I$. Obviously, we have that $a I \subseteq I$. Thus, $\forall c \in I$ and $\forall b \in J$ it holds that $a(I+J) \subseteq I$. Therefore, $a \in A n n((I+J) / I)$.

(iii). Let $a \in A n n(I)$. Then, by definition, $a \in F$ with $a I=0$ and since $I \cong V / J$ we have that $a \in F$ with $a(V / J)=0$. Thus, $a$ belongs to $J$.

Conversely, let $a$ be an element of $J$. It implies that $a(V / J)=0$ and since $M \cong V / J$ we have that $a I=0$. Thus, $a \in A n n(I)$.

\subsection{Localization}

Next we will see how we can create a local ring from an arbitrary integral domain. The main idea of creating a local ring is to transform an integral domain where not all elements are invertible into a local ring where we can determine exactly which elements are invertible.

Lemma 4.2.1. Let $P$ be a prime ideal of $V$. The set

$$
V_{P}=\left\{\frac{a}{b} \mid a \in V, b \in V-P\right\}
$$

is a local ring $V_{P}$ and

$$
P^{\prime}=\left\{\frac{a}{b} \mid a \in P, b \in V-P\right\}
$$

is its maximal ideal.

Proof. Every element $a / b$ of $V-P^{\prime}$ is a unit because if $a$ does not belong to $P$, then $b / a$ belongs to $V_{P}$. Obviously

$$
\frac{a}{b} \cdot \frac{b}{a}=1
$$

and therefore $a / b$ is a unit. So by the proposition 1.2.1 we get that $V_{P}$ is a local ring.

In order to prove that $P^{\prime}$ is a maximal ideal, assume that $a / b \in P^{\prime}$ and $c / d \in V_{P}$. Then

$$
\frac{a}{b} \cdot \frac{c}{d}=\frac{a c}{b d} \in P^{\prime}
$$


since $a c \in P$ and $b d \notin P$.

The procedure of obtaining the ring $V_{P}$ from $V$ is called localization of $V$ at $P$. Let

$$
P=\left\{p^{n} \mid p \in V\right\} \text {. }
$$

The corresponding localization

$$
V_{p}=\left\{\frac{a}{b} \mid a \in V, b \in V-P\right\}
$$

is called localization of $V$ at $p$. For example, if $V=\mathbb{Z}$ and $a=2$ we get

$$
\mathbb{Z}_{(2)}=\left\{\frac{a}{b} \mid a \in \mathbb{Z}, b \text { odd }\right\}
$$

with maximal ideal

$$
P_{(2)}=\left\{\frac{a}{b} \mid a \text { even }, b \text { odd }\right\} \text {. }
$$

Analogously to the definition of $V_{P}$, we define the set

$$
I_{P}=\left\{\frac{a}{b} \mid a \in I, b \in V-P\right\}
$$

to be the localized fractional ideal $I$ at $P$. One can see that if there is an $a \in V$ such that $a I \subset V$ it implies that $a I_{P} \subset V_{P}$. So, $I_{P}$ is a fractional ideal of $V_{P}$. The next two lemmata provide us with some basic properties of localized fractional ideals.

Lemma 4.2.2. Let $I$, $J$ be two fractional ideals and $P$ a prime ideal of $V$. It holds that

(i) $(I+J)_{P}=I_{P}+J_{P}$.

(ii) $(I J)_{P}=I_{P} J_{P}$.

(iii) $(I \cap J)_{P}=I_{P} \cap J_{P}$.

(iv) $(I / J)_{P} \cong I_{P} / J_{P}$.

Proof. During the proof $a, b, s, s_{1}$ and $s_{2}$ are elements such that $a \in I, b \in J$ and $s, s_{1}, s_{2} \notin P$.

(i) Let $(a+b) / s \in(I+J)_{P}$. It implies that $a / s+b / s \in I_{P}+J_{P}$, thus $(I+J)_{P}$ is a subset of $I_{P}+J_{P}$. Now let $a / s_{1} \in I_{P}$ and $b / s_{2} \in J_{P}$. It holds that

$$
\frac{a}{s_{1}}+\frac{b}{s_{2}}=\frac{a s_{2}+b s_{1}}{s_{1} s_{2}}
$$

Obviously, since $I, J$ and $S$ are ideals, $a s_{2}$ belongs to $I, b s_{1}$ belongs to $J$ and $s_{1} s_{2}$ does not belongs to $P$. Therefore, $\left(a s_{2}+b s_{1}\right) /\left(s_{1} s_{2}\right)$ belongs to $(N+M)_{P}$. The result follows.

(ii). The proof is analogous to (i).

(iii). Since $I \cap J \subset I$ it follows that $(I \cap J)_{P} \subset I_{P}$. Similarly, we know that $(I \cap J)_{P} \subset J_{P}$ and therefore

$$
(I \cap J)_{P} \subset I_{P} \cap J_{P} .
$$

Now let $x \in I_{P} \cap J_{P}$. Thus there are $a \in I, b \in J$ and $s_{1}, s_{2} \in P$ such that

$$
x=\frac{a}{s_{1}}=\frac{b}{s_{2}} .
$$


So

$$
y=a s_{2}=b s_{1} \in I \cap J
$$

and therefore

$$
x=y\left(s_{1} s_{2}\right)^{-1} \in I_{P} \cap J_{P}
$$

which implies that $I_{P} \cap J_{P} \subset(I \cap J)_{P}$.

(iv). For $J \subset I$ we introduce functions $\iota$ and $\sigma$ as follows

$$
\iota: I_{P} \rightarrow J_{P}, a / s \rightarrow b / s
$$

and

$$
\sigma: J \rightarrow(I / J), \quad b / s \rightarrow b / s .
$$

One can see that $\iota$ is an injection and that $\sigma$ is a surjection. Therefore, we have

$$
(I / J)_{P} \cong \operatorname{Im}(\sigma) \cong I_{P} / \operatorname{Ker}(\sigma) \cong I_{P} / \operatorname{Im}(\iota) \cong I_{P} / J_{P}
$$

The result follows.

Lemma 4.2.3. Let $I$, $J$ be fractional ideals and $P$ a prime ideal of $V$. It holds that

(i) $\operatorname{Ann}(J)_{P}=\operatorname{Ann}\left(J_{P}\right)$

(ii) $(I: J)_{P}=\left(I_{P}: J_{P}\right)$.

Proof. (i). We will prove this lemma inductively. Since the fractional ideals are finitely generated, we will first prove the lemma for the case where a fractional ideal is produced by a single element, and then for the case that we have a sum of fractional ideals.

Let $J$ be a fractional ideal generated by a single element initially. Then, its elements will be all elements of $V$ except those elements that vanish all elements of $J$. Therefore

$$
J \cong V / A n n(J)
$$

and by lemma 4.2.2 we get

$$
J_{P} \cong V_{P} / A n n(J)_{P}
$$

Finally, from the above conjugation by lemma 4.1.4 we get that

$$
\operatorname{Ann}\left(J_{P}\right)=\operatorname{Ann}(J)_{P}
$$

which means that the lemma holds for the case where the fractional ideal is generated by a single element. Next, we assume that the lemma holds for two fractional ideals $J_{1}$ and $J_{2}$. Thus, we have that

$$
\operatorname{Ann}\left(J_{i}\right)_{P}=\operatorname{Ann}\left(\left(U_{i}\right)_{P}\right)
$$

for $i \in\{1,2\}$. We will show that the same holds for $J_{1}+J_{2}$. Applying localization to lemma 4.1.4 we have that

$$
A n n\left(J_{1}+J_{2}\right)_{P}=\left(A n n\left(J_{1}\right) \cap A n n\left(J_{2}\right)\right)_{P}
$$


and by lemma 4.2.2(ii) we get

$$
\operatorname{Ann}\left(J_{1}+J_{2}\right)_{P}=A n n\left(J_{1}\right)_{P} \cap A n n\left(J_{2}\right)_{P} .
$$

Since we have assumed that the lemma holds for $J_{1}$ and $J_{2}$ we know

$$
\operatorname{Ann}\left(J_{1}+J_{2}\right)_{P}=\operatorname{Ann}\left(\left(J_{1}\right)_{P}\right) \cap \operatorname{Ann}\left(\left(J_{2}\right)_{P}\right) .
$$

Again, by 4.1.4(i) and 4.2.2(i) we get

$$
A n n\left(\left(J_{1}\right)_{P}\right) \cap A n n\left(\left(J_{2}\right)_{P}\right)=A n n\left(\left(J_{1}\right)_{P}+\left(J_{2}\right)_{P}\right)=A n n\left(\left(J_{1}+J_{2}\right)_{P}\right) .
$$

Therefore the lemma holds for any fractional ideal.

(ii). By lemma 4.1.4 we have that

$$
(I: J)=\operatorname{Ann}((I+J) / I)
$$

By localization we get that

$$
(I: J)_{P}=\operatorname{Ann}((I+J) / I)_{P}
$$

and as we have proved in (i)

$$
(I: J)_{P}=\operatorname{Ann}\left(((I+J) / I)_{P}\right) .
$$

By using now 4.2.2 we get

$$
\left.(I: J)_{P}=\operatorname{Ann}\left(\left(I_{P}+J_{P}\right) / I_{P}\right)\right)=\left(I_{P}: J_{P}\right) .
$$

The result follows.

Proposition 4.2.4. Let $I$ be a fractional ideal of $V$. If for every maximal ideal $P$ of $V$, $I_{P}$ is invertible then I is invertible.

Proof. Let $J=I \cdot(V: I)$. According to 4.1.1 in order to prove that $I$ is invertible it is enough to show that $J=V$. We localize the relation

$$
J=I \cdot(V: I)
$$

and by using 4.2.3 we get

$$
J_{P}=I_{P} \cdot\left(V_{P}: I_{P}\right) .
$$

Since $I_{P}$ is invertible we obtain that $J_{P}=V_{P}$. So, we have established that $J \not \subset P$ for every maximal ideal of $V$ and therefore $J=V$. 


\subsection{R-module V}

In this section we prove some properties of the $R$-module $V$.

Lemma 4.3.1. Let $V$ be a Noetherian integral domain and $F$ its field of fractions. $A$ nonzero $V$-submodule I of $F$ is a fractional ideal of $V$ if and only if it is finitely generated.

Proof. Suppose that $I$ is a fractional ideal of $V$. Then there is $a \in F$ such that $a I \subset V$. Since $V$ is Noetherian it is finitely generated, and therefore, $I$ is too.

Conversely, suppose $I$ is finitely generated and let $a$ be the least common multiple of the denominators of the generators of $I$. By multiplying $I$ with $a$ it turns to be a $V$-module which implies that $a I \subset V$ and therefore is fractional.

Lemma 4.3.2. Let $v \in V . R[v]$ is finitely generated as a R-module if and only if $v$ is integral over $R$.

Proof. Suppose that $R[v]$ is finitely generated. Every element $a \in R[v]$ can be written as

$$
a=\sum r_{i} v^{j}
$$

where $r_{i} \in R$, which is actually an $R$-linear combination of $v$. Therefore $v$ is integral over $R$.

Conversely, suppose that $v$ is integral over $R$. Then, there are $r_{i} \in R$ such that

$$
v^{n}+r_{n-1} v^{n-1}+\cdots+r_{1} v+r_{0}=0 \text {. }
$$

Thus,

$$
v^{n}=-r_{n-1} v^{n-1}-\cdots-r_{1} v-r_{0}
$$

which implies that $v^{n}$ belongs to the $R$-module generated by the set $\left\{1, v, \ldots, v^{n-1}\right\}$. For any rational integer $r>0$ we have that

$$
v^{n+r}=\left(-r_{n-1} v^{n-1}-\cdots-r_{1} v-r_{0}\right) v^{r} .
$$

Hence $R[v]$ is an $R$-module generated by $\left\{1, v, \ldots, v^{n-1}\right\}$.

Lemma 4.3.3. Let $V$ be a R-module that contains a submodule $N$ such that $V / N$ and $N$ are Noetherian $R$-modules. Then $V$ is a Noetherian $R$-module.

Proof. Let

$$
P_{1} \subset P_{2} \subset \cdots
$$

be an ascending sequence of $R$-submodules of $V$. Since $N \subset V$ we get that

$$
P_{1} \cap N \subset P_{2} \cap N \subset \cdots
$$


is also an ascending sequence of $R$-submodules of $V . N$ is Noetherian and therefore there is $s$ such that

$$
P_{S} \cap N=P_{s+1} \cap N=\cdots .
$$

Similarly, for ascending sequence

$$
\left(P_{1}+N\right) / N \subset\left(P_{2}+N\right) / N \subset \cdots
$$

of $V / N$, there is $t$, such that

$$
\left(P_{t}+N\right) / N=\left(P_{t+1}+N\right) / N=\cdots
$$

and therefore $N+P_{t}=N+P_{t+1}=\cdots$. Now let $p^{\prime} \in P_{t+1}$. Hence, $p^{\prime} \in N+P_{t+1}$ which implies that $p^{\prime} \in N+P_{t}$ and therefore $p^{\prime}=n+p$ for some $p \in P_{t}$ and $n \in N$. Thus

$$
n=p-p^{\prime} \in P_{t} \Rightarrow n \in N \cap P_{t}=N \cap P_{t+1} \text {. }
$$

Hence, $n \in P_{t}$ and since $p \in P_{t}$ it implies that $p^{\prime}=n+p \in P_{t}$. Thus

$$
P_{t}=P_{t+1}=\cdots
$$

which implies that $V$ is a Noetherian $R$-module.

Theorem 4.3.4. Let $R$ be a Noetherian ring. Every finitely generated $R$-module $V$ is Noetherian.

Proof. Assume that $V$ is generated by two elements $\left\{a_{1}, a_{2}\right\}$ which means that

$$
V=a_{1} R+a_{2} R
$$

Since $R$ is Noetherian it implies that $R_{i}=a_{i} R$ are Noetherian. Moreover, it holds that

$$
V / R_{1}=\left(R_{1}+R_{2}\right) / R_{1} \cong R_{2} /\left(R_{1} \cap R_{2}\right)
$$

Since $R_{1}$ and $R_{2}$ are Noetherian it implies that $R_{2} /\left(R_{1} \cap R_{2}\right)$ is Noetherian and therefore $V / R_{1}$ is Noetherian. Since $V / R_{1}$ and $R_{1}$ are Noetherian by 4.3 .3 we obtain that $V$ is Noetherian. The result follows inductively.

Obviously, the above theorem holds if $R$ is a principal ideal domain since by proposition 1.1.10 $R$ is Noetherian too. 